\title{
Conceptualization in Pakistani Bilinguals: A Cognitive Approach
}

\author{
Katsiaryna Hurbik \\ The University of Faisalabad, Faisalabad, Pakistan
}

\begin{abstract}
The paper is devoted to the representation of conceptual changes among Pakistani Urdu and English bilinguals. The aim is to analyze how Urdu as L1 influences cognition of basic concepts in English as L2. The research is based on qualitative analysis of casual discourse as well as quantitative analysis of a questionnaire containing only few verbal items. The sample was collected by stratifying the participants into age groups of $18-25,26-35,36-45$, 46-55, and 56-65 year old females and males of Lahore and Faisalabad. The examples of the casual discourse were taken both from Urdu and English. Combined levels of triangulation on the individual and interactive levels ensure validity and reliability of the research. The results revealed that not only Urdu as L1 has contributed to the change in conceptualization in English as L2, but English has modified cognition and perception of certain concepts in Urdu as well.
\end{abstract}

Keywords: concepts, bilingualism, conceptual change, Pakistani English (PE)

\section{Introduction}

The primary aim of the study is to analyze the changes in conceptualization among Pakistani Urdu and English bilinguals that occurred due to mutual influence of both languages and led to the emergence and wide recognition of Pakistani English as the variety of the English language.

In our case, we analyze changes in the English and Urdu languages that are not occasional or temporary, but have firmly settled among Pakistani bilinguals and serve as flag posts allowing identification of PE as a variety of English. The English language has been present in Pakistani society for almost 400 years. It has coexisted with other native languages of various provinces of the country that has resulted in a deep integration of English into all fields of social life. We intend to analyze how English modified the cognition of speakers to the extent allowing them conceptualize under the influence of the English language. On the other hand, we need to see whether the native Urdu has also transformed the basic concepts of the English language, accommodating them to the Pakistani society, adding culture-specific meanings and connotations to them.

We analysed basic frequently occurring concepts through their prior extraction from casual discourse using unstructured qualitative observation. The selected concepts then were included into the questionnaire containing few verbal items. Using both methods of data collection allowed us to reach validity of the research and understand conceptualization in Pakistani bilinguals through their cognition.

Katsiaryna Hurbik, assistant professor, Ph.D. scholar, Department of English Language and Literature, The University of Faisalabad. 


\section{Literature Review}

Cognitive approach to the study of language is different from others as it studies the way thoughts are formed in the mind of a language user and, consequently, conceptualized by the language users. As mentioned by Evans and Green (2006, p. 6), one crucial function of language is to express thoughts and ideas. That is, language encodes and externalizes our thoughts. The way language does this is by using symbols. The process of encoding ideas and thoughts may seem easy at the very first sight. Nevertheless, at a closer look it will be apparent that the process of conceptualization and forming of an idea in one's mind may encounter many obstacles while trying to convert it into words. The reason behind it is connected with the limitations that language sets to its users in expressing the thoughts and the fact that conceptualizing thoughts might have no limits, while the means of their language expression do.

First of all, it is necessary to mention that there is no unity on many basic categories and aspects among cognitive linguists. The theoretical body of the science has not been agreed on completely. Therefore, it is important to define the view we share on the main categories in the paper.

It is vital to start with the definition of the concept and conceptualization that is used in the context of the paper. Langacker (2008) proposed in his book that, in the first place, meaning is not identified with concepts but with conceptualization, the term being chosen precisely to highlight its dynamic nature. Conceptualization is broadly defined to encompass any facet of mental experience. It is understood as subsuming (1) both novel and established conceptions; (2) not just intellectual notions, but sensory, motor, and emotive experience as well; (3) apprehension of the physical, linguistic, social, and cultural context; and (4) conceptions that develop and unfold through processing time (rather than being simultaneously manifested). So even if "concepts" are taken as being static, conceptualization is not.

We also have to look into the structure of concepts in order to better understand the way concepts are structured and presented in a person's mind. As there are two different opinions on the structure and the nature of concepts: propositional and imagistic, we will rely on imagistic character of concepts. As proposed by Langacker (2008, p. 30) the best-known proposal posits a set of image schemas, described as schematized patterns of activity abstracted from everyday bodily experience, especially pertaining to vision, space, motion, and force. Even though many researchers agree that people structure categories by schemas, it is often difficult to find common features that display the membership to a certain category and its degree.

Another aspect relevant for the paper is the way language tends to reflect the ability of a person to express oneself in the society, to pass the idea to the others and make them understand it. It is only possible in case if the other people share the same knowledge and conceptualize in the similar, if not the same, way. Referring to Evans and Green (2006, p. 11), not only does language encode particular meanings, but also that, by virtue of these meanings and the forms employed to symbolize these meanings which constitute part of shared knowledge in a particular speech community, language can serve an interactive function, facilitating and enriching communication in a number of ways.

Langacker (2008) shares the definition regarding the meanings and the sources they are derived from for the cognitive approach, offering the interactive alternative which does take people into account but claims that an individual mind is not the right place to look for meanings. Instead, meanings are seen as emerging dynamically in discourse and social interaction. Rather than being fixed and predetermined, they are actively 
negotiated by interlocutors on the basis of the physical, linguistic, social, and cultural context. Meaning is not localized but distributed, aspects of it inhering in the speech community, in the pragmatic circumstances of the speech event, and in the surrounding world. In particular, it is not inside a single speaker's head.

The above mentioned points established the design of the research and underpinned the choice of methods.

\section{Methodology}

A multi-method approach has been selected for analyzing the process of conceptualization in Pakistani bilinguals. Langacker's (1987) imagery method of analyzing concepts was put as a base of the paper. The term imagery,

Indicates our undeniable capacity to construe a cognitive domain in alternate ways. Various dimensions of imagery must be accommodated, the most significant being what I call the profile/base distinction. The base for a linguistic predication is its domain, i.e., the cognitive structures it presupposes; its profile is a substructure of the base that is elevated to the distinctive level of prominence as an entity which the expression designates. Expressions often evoke the same domain, but contrast semantically by choosing alternate profiles within the common base. (Langacker, 1987, p. 56)

What we are interested in our paper is the scope identified by Langacker $(1987$, p. 56) as the scope of a predication is that portion of relevant domains which it specifically invokes and requires for its characterization.

The paper presents a small-scale research with the data collected in two stages. The sample was collected by stratifying the participants into age groups of 18-25, 26-35, 36-45, 46-55, and 56-65 year old females and males of Lahore and Faisalabad and consisted of 1,000 participants. At stage one, an unstructured qualitative observation of a casual discourse of the bilinguals was conducted. The choice of the unstructured type of observation is predefined by the fact that the data from the observation appears naturally, without prior knowledge of what key concepts will be derived during the process. It led us to getting a rich data from various situations and generation of the hypothesis.

The qualitative approach to data analysis was selected for the first stage of the research. Our aim was not to calculate quantitative occurrences of various concepts, but rather to make sense of data in terms of the participants' definitions of the situation, noting patterns, themes, categories, and regularities, as stated by Cohen, Manion, and Morrison (2007, p. 461). Systematic approach to the analysis of the observation was used. We implemented tactics suggested by Miles and Huberman (1994) that analyzes qualitative data moving from the specific to the general.

The second stage of the data collection was conducted by the questionnaire with non-verbal items. The concepts extracted from the observation were included for the purpose of the verification of ways of their cognition and conceptualization through prototypes. It contained visual information in the form of pictures and the respondents were asked to name the objects visualized. To increase the level of comprehension of the respondents of what they saw, the size of the picture was adjusted to fit full A4 paper with one picture per paper and a colored high quality picture was used. It helped to eliminate ambiguity as for the object displayed. For the second stage of the research, the variable was changed. The respondents were stratified on the basis of level of education: intermediate, graduate, and postgraduate (Master's and Doctorate). At the stage two, the data was analyzed quantitatively by using exploratory data analysis: cross tabulation by totals.

Reliability and validity of the research was ensured by combined levels of triangulation. On one hand, it ensured that our observations regarding cognition of the basic concepts and their conceptualization was correct; 
on the other hand, questionnaire conducted among respondents that were not the part of the observation allowed us to verify our hypothesis and also support it with further details.

\section{Results}

The aim of the first stage was to analyze a casual discourse by conducting a non-participant observation of Pakistani bilinguals for the purpose of extracting "live" data and making initial hypothesis regarding the cognition of basic and common concepts and the way they are conceptualized through prototypes. The results revealed that among common and basic concepts occurring in a casual discourse are prayer, dress, suit, and shopper. The following concepts were further focused upon.

Table 1 below illustrates the qualitative results of the observation of the selected concepts for conceptualization based on prototypes.

Table 1

Summary of Conceptualization Among Pakistani Bilinguals

\begin{tabular}{|c|c|c|c|}
\hline Age Concept & Prayer & Dress & Shopper \\
\hline $18-25$ & $\begin{array}{l}\text { Solemn request } \\
\text { Regular religious service }\end{array}$ & $\begin{array}{l}\text { Male clothing/garments } \\
\text { Formal attire }\end{array}$ & Plastic bag for shopping \\
\hline $26-35$ & $\begin{array}{l}\text { Solemn request } \\
\text { Regular religious service }\end{array}$ & $\begin{array}{l}\text { Male clothing/garments } \\
\text { Formal attire }\end{array}$ & Plastic bag for shopping \\
\hline $36-45$ & $\begin{array}{l}\text { Solemn request } \\
\text { Regular religious service }\end{array}$ & $\begin{array}{l}\text { Male clothing/garments } \\
\text { Female shalwar kameez }\end{array}$ & Plastic bag for shopping \\
\hline $46-55$ & Regular religious service & $\begin{array}{l}\text { Male clothing/garments } \\
\text { Female shalwar kameez }\end{array}$ & Plastic bag for shopping \\
\hline $56-65$ & Regular religious service & $\begin{array}{l}\text { Garments } \\
\text { Female shalwar kameez }\end{array}$ & Plastic bag for shopping \\
\hline
\end{tabular}

The analysis showed that the conceptualization of the basic concepts is similar in all age groups. It was observed that bilinguals tend to use the word prayer to express the ideas connected with both offering a regular five-time religious service as well as addressing God for requests. Although, a tendency to use the word prayer for offering a regular service only for conceptualization among bilinguals of 56 to 65 years of age was revealed. A strong influence of culture is revealed in conceptualization of dress. Culture-specific meanings and connotations were discovered after analyzing the discourse among all age groups of bilinguals: dress is used to describe clothes or formal attire of both females and males. Conceptualization of the concept shopper displayed the same conceptualization in all observed age groups used by all bilinguals as a plastic bag.

Results of quantitative analysis of the concepts displayed the influence of the educational level of respondents on cognition. It was revealed that the bilinguals with intermediate level of education do not conceptualize prayer as a dual act, but tend to think of it as a regular service they offer. On the contrary, the respondents of Ph.D. level did not think of prayer as a solemn request rather considering both ideas. The dominating $58.3 \%$ in the meaning of the concept among the respondents stating it to be a regular service may be explained by the fact that there exist two different words in Urdu to conceptualize the regular religious service as namaz and dua is used to express a solemn request. Namaz and dua are conceptualized differently as the following concepts have different meanings in Urdu. It usually creates confusion in expressing the idea in English due to the absence of equivalents in the English language. As a result, code-switching usually occurs in such cases (see Table 2). 
Table 2

Cross-Tabulation by Totals of the Concept "Prayer"

\begin{tabular}{|c|c|c|c|c|c|c|}
\hline \multicolumn{7}{|l|}{ Prayer } \\
\hline & & & $\begin{array}{l}\text { Regular religious } \\
\text { service }\end{array}$ & Solemn request & Both & Total \\
\hline \multirow{2}{*}{\multicolumn{2}{|c|}{ Intermediate }} & Count & 381 & 75 & - & 456 \\
\hline & & $\%$ of total & $38.1 \%$ & $7.5 \%$ & - & $45.6 \%$ \\
\hline \multirow{2}{*}{ Graduate } & & Count & 143 & 102 & 69 & 314 \\
\hline & & $\%$ of total & $14.3 \%$ & $10.2 \%$ & $6.9 \%$ & $31.4 \%$ \\
\hline \multirow{4}{*}{$\begin{array}{l}\text { Post } \\
\text { graduate }\end{array}$} & \multirow{2}{*}{ Master's } & Count & 56 & 16 & 132 & 204 \\
\hline & & $\%$ of total & $5.6 \%$ & $1.6 \%$ & $13.2 \%$ & $20 \%$ \\
\hline & \multirow{2}{*}{ Ph.D. } & Count & 3 & - & 23 & 26 \\
\hline & & $\%$ of total & 0.3 & - & 2.3 & $3 \%$ \\
\hline \multirow{2}{*}{\multicolumn{2}{|c|}{ Total }} & Count & 583 & 193 & 224 & 1,000 \\
\hline & & $\%$ of total & $58.3 \%$ & $19.3 \%$ & $22.4 \%$ & $100 \%$ \\
\hline
\end{tabular}

The results of analysis of the concept dress demonstrated that the majority of bilinguals of intermediate and graduate educational level conceptualize it as male clothing/garments and also associate it with female shalwar kameez (see Table 3).

Table 3

Cross-Tabulation by Totals of the Concept "Dress"

\begin{tabular}{|c|c|c|c|c|c|c|}
\hline \multicolumn{7}{|l|}{ Dress } \\
\hline & & & Garments/Clothing & Female shalwar kameez & Formal attire & Total \\
\hline \multirow{2}{*}{\multicolumn{2}{|c|}{ Intermediate }} & Count & 313 & 111 & 32 & 456 \\
\hline & & $\%$ of total & $31.2 \%$ & $11.2 \%$ & $3.2 \%$ & $45.6 \%$ \\
\hline \multirow{2}{*}{\multicolumn{2}{|c|}{ Graduate }} & Count & 209 & 68 & 37 & 314 \\
\hline & & $\%$ of total & $20.9 \%$ & $6.8 \%$ & $3.7 \%$ & $31.4 \%$ \\
\hline \multirow{4}{*}{$\begin{array}{l}\text { Post } \\
\text { graduate }\end{array}$} & \multirow{2}{*}{ Master's } & Count & 68 & 112 & 24 & 204 \\
\hline & & $\%$ of total & $6.8 \%$ & $11.2 \%$ & $2.4 \%$ & $20 \%$ \\
\hline & \multirow{2}{*}{ Ph.D. } & Count & 6 & 17 & 3 & 26 \\
\hline & & $\%$ of total & $0.6 \%$ & $1.7 \%$ & $0.3 \%$ & $3 \%$ \\
\hline \multirow{2}{*}{\multicolumn{2}{|c|}{ Total }} & Count & 596 & 308 & 96 & 1,000 \\
\hline & & $\%$ of total & $59.6 \%$ & $30.8 \%$ & $9.6 \%$ & $100 \%$ \\
\hline
\end{tabular}

The reason behind the way Pakistani bilinguals conceptualize lies in absence of equivalents of separate garment items such as trousers, T-shirt, and shirt in Urdu. Shalwar kameez is Pakistani traditional clothing and is usually called collectively as garments or clothes. So, when it comes to expressing the idea in English, bilinguals use the word dress, meaning different types of garments, including shalwar kameez, worn by males and females. With the respondents of graduate and postgraduate level, the greater percentage tends to conceptualize it as female shalwar kameez. The reason is the intention to indicate the difference between traditional clothing usually worn by females and other types of clothing worn mostly by males.

Analysis of the concept shopper acquired an additional meaning and was conceptualized by majority of bilinguals as a plastic shopping bag (see Table 4).

The respondents of intermediate, graduate, and postgraduate level demonstrated a complete absence of the option in conceptualizing it as a person doing shopping. It may be explained by zero equivalence of the concept 
in Urdu for the plastic bag used for shopping. The respondents of postgraduate level of education has added the meaning of a person doing shopping in their conceptualization, nevertheless adding the usage of the word for the shopping bag due to the zero equivalence in Urdu for this specific meaning.

Table 4

Cross-Tabulation by Totals of the Concept "Shopper"

\begin{tabular}{|c|c|c|c|c|c|c|}
\hline \multicolumn{7}{|l|}{ Shopper } \\
\hline & & & $\begin{array}{l}\text { Plastic bag used for } \\
\text { shopping }\end{array}$ & $\begin{array}{l}\text { A person who is } \\
\text { shopping }\end{array}$ & Both & Total \\
\hline \multirow{2}{*}{\multicolumn{2}{|c|}{ Intermediate }} & Count & 456 & - & - & 456 \\
\hline & & $\%$ of total & $45.6 \%$ & - & - & $45.6 \%$ \\
\hline \multirow{2}{*}{ Graduate } & & Count & 314 & - & - & 314 \\
\hline & & $\%$ of total & $31.4 \%$ & - & - & $31.4 \%$ \\
\hline \multirow{4}{*}{$\begin{array}{l}\text { Post } \\
\text { graduate }\end{array}$} & \multirow{2}{*}{ Master's } & Count & 125 & 63 & 16 & 204 \\
\hline & & $\%$ of total & $12.5 \%$ & $6.3 \%$ & $1.6 \%$ & $20 \%$ \\
\hline & \multirow{2}{*}{ Ph.D. } & Count & - & 5 & 21 & 26 \\
\hline & & $\%$ of total & - & $0.5 \%$ & $2.1 \%$ & $3 \%$ \\
\hline \multirow{2}{*}{ Total } & & Count & 895 & 68 & 37 & 1,000 \\
\hline & & $\%$ of total & $89.5 \%$ & $6.8 \%$ & $3.7 \%$ & $100 \%$ \\
\hline
\end{tabular}

Analyzing the data in two steps by using qualitative and quantitative methods allowed us to achieve accuracy in results. Changing the variables enabled us to answer the question set in the beginning of the research and discover the way Pakistanis conceptualize in the bilingual setup.

\section{Conclusion}

The aim of the study was to analyze what influence English has on the cognition processes of bilinguals as well as the impact Urdu has on English in the language situation among its users.

The findings demonstrate that conceptualization of Pakistani bilinguals is heavily influenced by the use of the English language in many spheres of life and resulted in forming of certain concepts in the cognition of the bilinguals that have zero equivalence in Urdu. On the other hand, Urdu has added more meanings to English, specific to the Pakistani culture.

The limitation of the study was the geography of the sample as the population was taken from Faisalabad and Lahore only. Larger sample size may also have contributed to the results of the research. Another limitation is connected with the time taken for the observation. More concepts with explicit meanings could be discovered if the population were observed for a more prolonged time.

The results have proved the need for further analysis in the field of cognitive study in Pakistani bilinguals that will contribute in systemizing and structurizing of the body of Pakistani variety of the English language.

The results may prove useful for the researchers in the field of PE and other varieties of English of the world, providing more insights into the studies of socio-cultural, cognitive, and psychological aspects of the language. It will also help educationalists and translators in better understanding and interpretation of concepts.

\section{References}

Baumgardner, R. (1993). The English language in Pakistan. Karachi: The Oxford Press.

Cohen, L., Manion, L., \& Morrison, K. (2007). Research methods in education. London \& New York: Routledge.

Evans, V., \& Green, M. (2006). Cognitive linguistics. Edinburgh: Edinburgh University Press. 
Hartford, B., \& Mahboob, A. (2004). Models of discourse. World Englishes, 23, 585-600.

Kachru, B. B. (1982). The other tongue. Oxford: Pergamum.

Langacker, R. W. (1987). Nouns and verbs. Language, 63, 53-94.

Langacker, R. W. (2008). Cognitive grammar: A basic introduction. New York: Oxford University Press.

Mahboob, R. (2009). English as an Islamic language: A case study of Pakistani English. World Englishes, 28, 175-189.

Pennycook, A. (1998). English and the discourses of colonialism. London: Routledge.

Rahman, T. (1990). Pakistani English. Islamabad: National Institute of Pakistan Studies Quaid-e-Azam University.

Rosch, E. H., Mervis, C. B., Gray, W. D., Johnson, D. M., \& Boyes-Braem, P. (1976). Basic objects in natural categories.

Cognitive Psychology, 8, 382-439. 